\title{
Towards a realistic 3D simulation of the extraction region in ITER NBI relevant ion source
}

\author{
S. Mochalskyy, ${ }^{1}$ D. Wünderlich, ${ }^{1}$ U. Fantz, ${ }^{1}$ P. Franzen ${ }^{1}$ and T. Minea ${ }^{2}$ \\ ${ }^{1}$ Max-Planck-Institut für Plasmaphysik, Boltzmannstr. 2, D-85748, Garching, Germany \\ ${ }^{2}$ Laboratorire de Physique des Gaz et des Plasmas, CNRS, University Paris-Sud, 15 rue G. Clemenceau, F91405, Orsay, \\ France
}

\section{Abstract}

The development of negative ion sources for ITER is strongly accompanied by modelling activities. The ONIX code addresses the physics of formation and extraction of negative hydrogen ions at ceasated sources as well as the amount of co-extracted electrons. In order to be closer to the experimental conditions the code has been improved. It includes now the bias potential applied to first grid (plasma grid) of the extraction system, and the presence of $\mathrm{Cs}+$ ions in the plasma. The simulation results show that such aspects play an important role for the formation of an ion-ion plasma in the boundary region by reducing the depth of the negative potential well in vicinity to the plasma grid that limits the extraction of the negative ions produced at the Cs covered plasma grid surface. The influence of the initial temperature of the surface produced NI and its emission rate on the NI density in the bulk plasma that in turn affects the beam formation region was analyzed. The formation of the plasma meniscus, the boundary between the plasma and the beam, was investigated for the extraction potentials of $5 \mathrm{kV}$ and $10 \mathrm{kV}$. At the smaller extraction potential the meniscus moves closer to the plasma grid but as in the case of $10 \mathrm{kV}$ the deepest meniscus bend point is still outside of the aperture. Finally, a plasma containing the same amount of NI and electrons $\left(\mathrm{n}_{\mathrm{H}-}\right.$ $=\mathrm{n}_{\mathrm{e}}=10^{17} \mathrm{~m}^{-3}$ ), representing good source conditioning, was simulated. It is shown that at such conditions the extracted NI current can reach values of $\sim 32 \mathrm{~mA} / \mathrm{cm} 2$ using ITER relevant extraction potential of $10 \mathrm{kV}$ and $\sim 19 \mathrm{~mA} / \mathrm{cm} 2$ at $5 \mathrm{kV}$. These results are in good agreement with experimental measurements performed at the small scale ITER prototype source at the test facility BATMAN.

\section{Introduction}

The future fusion experimental reactor ITER will be equipped with two neutral beam injectors that will supply 33MW total power with beam energy of $1 \mathrm{MeV}$ during a pulse length of 3600s [1] [2]. As the cross-section for neutralization of positive hydrogen and deuterium ions at this energy is negligible low, a system based on the neutralization of negative ions has to be used. To reach ITER requirements the negative ion source should deliver $69 \mathrm{~A}$ extracted current in hydrogen and 57A in deuterium operation. Compared to positive hydrogen ion sources the current densities obtainable in negative ion sources are about 10 times lower. For ITER NBI $\mathrm{j}_{\mathrm{H}-}=34.5 \mathrm{~mA} / \mathrm{cm}^{2}$ and $\mathrm{j}_{\mathrm{D}-}=28.5 \mathrm{~mA} / \mathrm{cm}^{2}$ are planned. Therefore, the extraction area will be large $\left(\mathrm{A}_{\text {extr.ITER }} \approx 0.2 \mathrm{~m}^{2}\right)$ including 1280 apertures with a diameter of $14 \mathrm{~mm}$ each. In order to keep the stripping losses (negative ion destruction by collision with the background gas) in the extraction region and accelerator reasonably low, the filling pressure in the plasma source must be equal or below 0.3Pa. Negative ion extraction from a plasma intrinsically implies the co-extraction of electrons. These electrons are dumped onto the extraction grid (EG) preventing thus their full acceleration, by means of a deflection magnetic field, which is produced from permanent magnets embedded into the EG. To avoid melting and damaging of this grid due to a too high power load [3], the electron current density must be reduced to a tolerable value $\left(\mathrm{j}_{\mathrm{e}} \leq \mathrm{j}_{\mathrm{NI}}\right.$ for ITER design with $\left.\mathrm{U}_{\mathrm{ext}} \leq 10 \mathrm{kV}\right)$.

Limiting the co-extracted electron current and simultaneously improving the NI extraction is an important issue for NBI that involves deep study of the extraction region. Moreover, the meniscus shape (the region, where the extraction beam is separated from the plasma and the system potential $\approx 0 \mathrm{~V}$ ) and its position are the central parameters for the beam formation and the beam optic [2]. Realistic numerical simulations will help not only to improve our knowledge on the sheath (the 
region, where charge separation appears) but give hints for the further source optimization by understanding the processes governing the meniscus formation and the influence of plasma and source parameters on its position and shape.

A Radio-Frequency $(\mathrm{RF})$ driven $\left(\mathrm{P}_{\mathrm{RF}}<100 \mathrm{~kW}, \mathrm{f}_{\mathrm{RF}} \sim 1 \mathrm{MHz}\right)$ negative ion plasma source prototype for ITER NBI has been developed at IPP Garching - Fig. 1 [4] [5]. It consists of three main parts: the driver, the expansion chamber and the extraction system. A high temperature plasma $\left(\mathrm{T}_{\mathrm{e}} \approx 10 \mathrm{eV}, \mathrm{n}_{\mathrm{e}} \approx 10^{18} \mathrm{~m}^{-3}\right)$ is generated in the driver. The plasma then diffuses out from the driver into the expansion chamber where the electrons are cooled to a temperature of $\sim 1 \mathrm{eV}$ by means of a magnetic filter field, which is generated via permanent magnets installed upstream of the plasma grid (or by a current flowing through the plasma grid in larger sources and for ITER [6]). The extraction system consists of 3 multi-aperture grids: the plasma grid (PG), the extraction grid (EG) and the grounded grid (GG). A positive extraction voltage of $5-10 \mathrm{kV}(\sim 10 \mathrm{kV}$ for ITER) is applied between the plasma grid and the extraction grid to attract negative ions and repel positively charged particles.

The co-extracted electrons are one of the main limitations of the source performance. As it was mentioned above, these electrons are cooled down first by means of the magnetic filter field reducing also electron density by one order of magnitude and then dumped on the surface of the EG by means of the deflecting magnetic field, which is perpendicular to the filter field (Fig. 1 inset) and alternating from aperture to aperture. Further reduction can be obtained by a positive bias applied at the plasma grid against the bias plate which is connected to the source body [7]. Due to the complex inhomogeneous magnetic field distribution [4] [8] - breaking the symmetry of the system - only 3D models can perform calculation representing entirely the experimental conditions.

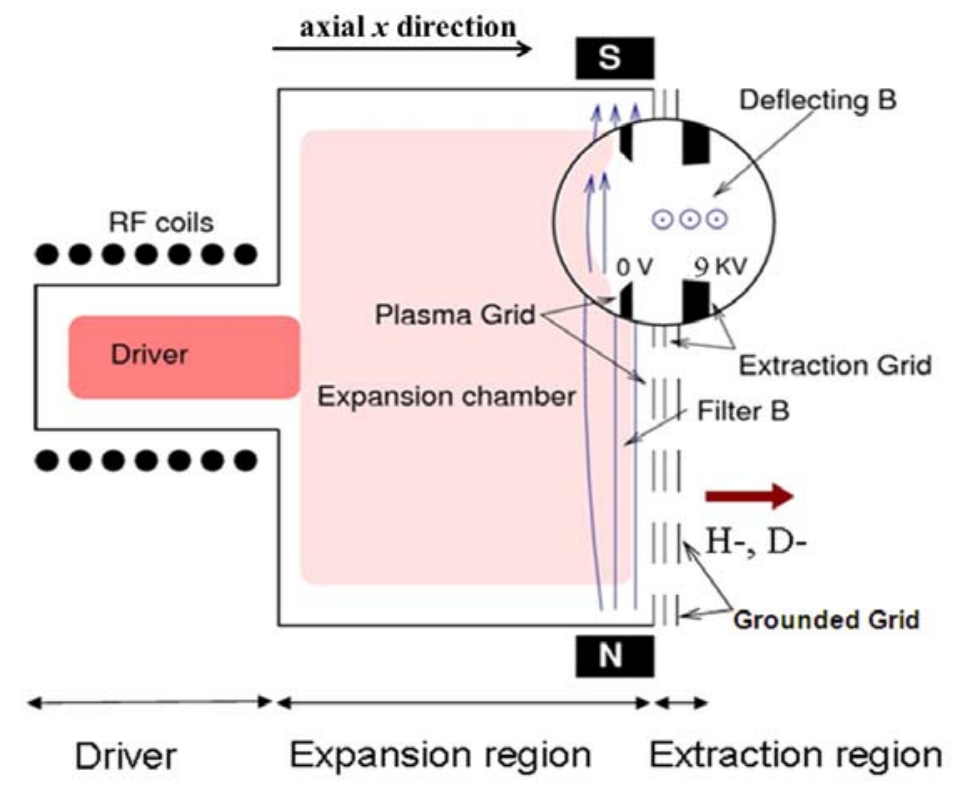

Fig. 1. Schematic view of the ITER NBI negative ion plasma source prototype designed at IPP. The $x$-axis corresponds to the axial beam direction while the $y$ (horizontal) and $z$ (vertical) axis is in the plane of the extraction grid.

Most of the ITER requirements were fulfilled by the RF driven prototype source, which is equipped with one driver ( 8 are planned for ITER) developed at the Max-Planck-Institut fuer Plasmaphysik (IPP), Garching, Germany [4] [5]. The test facility BAvarian Test MAchine for Negative ions (BATMAN) uses an extraction area $\mathrm{A}_{\text {ext.,BATMAN }} \approx \times 10^{-3} \mathrm{~m}^{2}$. The machine was dedicated to demonstrate the possibility to meet ITER requirements in terms of the extracted NI current density with simultaneously low $(<1)$ e/NI current ratio for the relevant source pressure during short plasma pulse length $(<6 \mathrm{~s})$. All these conditions have been successfully attained for both hydrogen and deuterium plasmas [4]. The Multi Ampere Negative Ion Test Unit (MANITU) is - similar to BATMAN - equipped with the IPP prototype source - and was built to demonstrate the feasibility to reach ITER requirements for long pulses (3600s) [9]. ELISE (Extraction from a 
Large Ion Source Experiment) [6] [10] [11] [12] [13] [14] is equipped with 4 drivers and it is now in operation at IPP. The testbed has been constructed to exhibit ITER NI source parameters for a large extraction area $\left(\mathrm{A}_{\text {ext.,ELISE }} \approx 0.1 \mathrm{~m}^{2}\right)$ and show a beam homogeneity with spatial and temporal variation below $10 \%$. Being close to these experimental testbeds allows performing simulations with relevant input data and include in the developed model appropriate source and plasma parameters.

Negative ions in these ion sources are born by two paths: volume and surface related processes, whereas in a good conditioned source the latter dominates by a factor of 10 . In the plasma in the expansion chamber the negative ions are created via dissociative electron attachment collisions to hydrogen molecules in high vibrational states $\left(\mathrm{H}_{2}(v)+\mathrm{e} \rightarrow \mathrm{H}^{+} \mathrm{H}^{-}\right)$[15]. However, the amount of the created NIs is low due to the low pressure and relatively low electron density. Moreover, most of these negative ions produced in the volume are destroyed there due to the variety of collisional processes [15] [16]. In order to enhance the amount of produced and extracted NI, caesium vapour is injected in the source [4] [17] [18]. Caesium covers the inner surface of the ion source including the plasma grid and NI are produced via surface conversion of impinging atoms $\left(\mathrm{H}+\mathrm{e} \rightarrow \mathrm{H}^{-}\right)$and positive ions $\left(\mathrm{H}^{+}+\mathrm{e} \rightarrow \mathrm{H} ; \mathrm{H}+\mathrm{e} \rightarrow \mathrm{H}^{-}\right)$.

It is known from the simulations that an important amount of the negative ions produced at the plasma grid surface are pushed back towards the plasma grid wall by means of the negative potential well (virtual cathode) [19] [20] [21] [22] [23] [24], which is formed from the space charge of the surface created NIs. Each of the available models used to describe this effect shows a different strength of the reflecting field due to the different depths of the negative potential well (from -0.5 to $-30 \mathrm{~V}$ ). Only one $1 \mathrm{D}$ code [21] for RF source, in spite of the virtual cathode formation, shows good agreement with experiments in terms of the negative ion density in the bulk plasma region $\left(\sim 10^{17} \mathrm{~m}^{-3}[25]\right)$ creating a so-called ion-ion plasma (a plasma in which negative ions contribute more to the total negative charge than the electrons $-n_{H-} \geq n_{e}$ [25] [26] [27]). The relatively high NI density in the bulk plasma was obtained also by 2D code for the arc source [20]. In other models the negative ions density is too low. One of the goals of this paper is to show the influence of different plasma and source parameters on the reduction of the depth of the potential well increasing thus the negative ion density in the bulk plasma region, which in turn affects the meniscus shape.

During the last years significant progress in the numerical modelling of the negative ion source was achieved. Different codes [19] [20] [21] [23] [24] [28] [29] [30] [31] [32] [33] for simulation of the plasma properties and negative ion extraction from RF and arc sources were developed and improved. However, the influence of the bias potential on the negative ion transport from the plasma grid surface towards the bulk plasma region was not studied with presence of the extraction potential. Moreover, the presence of positive $\mathrm{Cs}^{+}$[34] [35] ions in the plasma was previously considered only in a 1D PIC code [21]. The existence of heavy positively charged ions close to the plasma grid surface could reduce the depth of the negative potential well. Therefore, more negative ions will be able to overcome it for the same source conditions.

The 3D PIC MCC code ONIX [8] was improved in order to be closer to the experimental conditions and test new phenomena. It includes now the plasma grid bias potential and $\mathrm{Cs}^{+}$ions. Parametric study of these quantities were performed in order to check their influence on the meniscus formation, reduction of the negative potential well and negative ion density variation in the plasma bulk region. The simulated results in terms of the extracted NI and co-extracted electron current were benchmarked against the experimental measurement performed at the BATMAN testbed for both so called well and poor Cs conditioning representing source performance for high and low negative ion production rate at the plasma grid surface, respectively. The conditioning status in turn has an effect on the stability of the source performance and extracted NI and electron current [4].

The structure of this paper is as follows: section II describes the numerical features of the ONIX code, whereas the obtained results are presented and discussed in Section III. The last section summarizes the conclusions of this work and describes future plans. 


\section{Simulation model}

The 3D Particle-in-Cell Monte Carlo Collision code ONIX (Orsay Negative Ion eXtraction) has been initially developed in the laboratory LPGP, Orsay, France to simulate plasma behavior in the extended boundary layer (the plasma volume in the vicinity of the $\mathrm{PG}$, axial extent several $\mathrm{cm}$ ) of an ITER-like negative ion source extraction system. A detailed description of the code can be found elsewhere [8] [22] [30]. In this section only the most recent improvements of the code will be discussed, recalling briefly the general code properties.

ONIX can calculate both the extracted electron and negative ion currents and determines the origins of the extracted particles: (i) NI produced in the plasma volume; (ii) NI produced at the conical part of the plasma grid - Fig. 2 and (iii) NI produced at the flat part of the plasma grid. The code provides also the density distribution of electrons, positive and negative ions making possible to study the effect of the ion-ion plasma on the extracted negative species. The code is able to model both $\mathrm{H}^{-}$and $\mathrm{D}^{-}$ions, however for this paper only $\mathrm{H}^{-}$ions were simulated.

Typical runs are performed with a few $10^{6}$ macro particles (each representing $5 \times 10^{4}$ real particles) reducing thus the computation time, but simultaneously preserving the microscopic description of the phenomena. The charge of the macro particles is linearly interpolated and distributed onto the PIC nodes $(186 \times 70 \times 70$ in axial $x$, horizontal $y$ and vertical $z$ direction respectively) with the following dimensions of the mesh: $\Delta x=0.15 \mathrm{~mm}, \Delta y=\Delta z=0.2 \mathrm{~mm}$. The charge is further used as source term in Poisson's equation, which solution gives the electric field acting on the particles during the next time step. In this sense, ONIX is self-consistent.

For stable plasma PIC simulation few calculation conditions should be satisfied. The chosen time step must be smaller than the inverse plasma frequency $\Delta t=3 \cdot 10^{-12} s<\frac{1}{\omega_{p}}=3.97 \cdot 10^{-11} \mathrm{~s}$. The simulation PIC mesh size should be in a range (or smaller) than the Debye Length. In our model the mesh size $(\Delta x=0.15 \mathrm{~mm})$ is a few times larger than the Debye length $\left(\lambda_{D} \approx 0.026 \mathrm{~mm}\right)$ in the bulk plasma (expansion chamber), but only for the denser plasma region. However, the plasma density drastically drops close to the PG and Debye length condition $\left(\lambda_{D} \approx 0.15 \mathrm{~mm}\right)$ is completely fulfilled in the region of interest $(\sim 1 \mathrm{~cm}$ from the PG wall), namely the sheath including the meniscus. This assumption was tested by comparison of the calculation results with a smaller mesh size $\Delta x=0.1 \mathrm{~mm}$ and the identical results proved the initial choice. No significant differences could be revealed in terms of the extracted electron and NI currents as well as in the overall 3D potential distribution. The last and the most important criterion for the stable PIC simulation is the Courant-Friedrichs-Lewy (CFL) condition [36]. Under this requirement the particles with the maximum possible energy must not jump over one PIC cell, i.e. they must be present in each cell at least once per time step for each update of the potential map. For the chosen time step and size of the chosen PIC mesh this criterion is strictly respected in all the volume.

The code uses a Cartesian coordinate system and includes only one (central) aperture from the plasma grid. The simulation domain (Fig. 2) represents a typical aperture of the LAG (Large Area Grid) extraction system installed at the BATMAN testbed [4], allowing thus the direct comparison between numerical and experimental results. The size of the LAG plasma grid aperture is different from the ITER design: $8 \mathrm{~mm}$ diameter (14mm for ITER grid), $4 \mathrm{~mm}$ width (9mm for ITER grid) and double chamfered edges with the angle of 45 degree from each side [2] [4]. The distance from the plasma grid to the extraction grid is $3.5 \mathrm{~mm}$ (6mm for ITER [2]). The optimum perveance, the perveance being defined as $P=I_{e x} / U_{e x}^{3 / 2}$, where $I_{e x}$ is the total extracted current and

$U_{e x}$ the applied extraction voltage, for the LAG grid and an extracted NI current density $\sim 20 \mathrm{~mA} / \mathrm{cm}^{2}$ lies at an extraction potential of $\sim 5 \mathrm{kV}$ [37]. For the ITER-like grid configuration and ITERrelevant NI current densities the optimum perveance is at the extraction potential value $\sim 10 \mathrm{kV}$. The perveance defines also the beam quality resulting in the minimum divergence at the optimum perveance condition. 


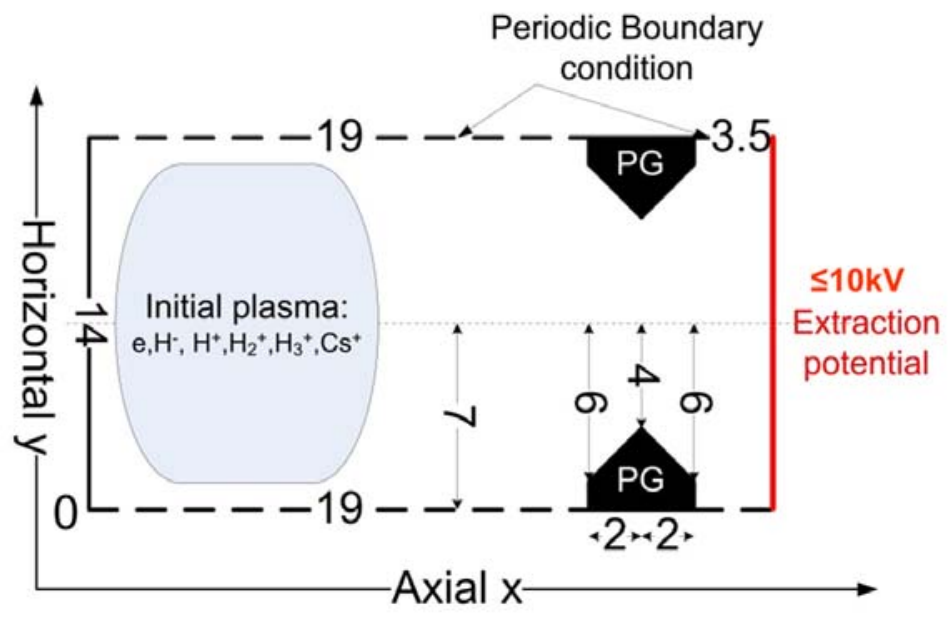

Fig. 2. Schematic view of the simulation domain used in ONIX. Units are shown in mm.

In our previous study [8] 9kV extraction potential was used for the LAG extraction system. This condition represents a high extracted NI current but bad perveance with poor beamlet optics. In the simulations for the current paper both the optimal $(5 \mathrm{kV})$ and poor perveance with maximum current $(\sim 10 \mathrm{kV})$ extraction potential were used in order to directly compare the simulation results with available experimental data for both conditions.

Periodic boundary conditions are used in horizontal $y$ and vertical $z$ directions representing an infinite $2 \mathrm{D}$ array of $\mathrm{PG}$ apertures. The extraction potential is applied to the right boundary of the domain, while the left hand side boundary is taken $0 \mathrm{~V}$.

The simulations were performed as close as possible to the experimental parameters using measured quantities as an input for the code (Table 1) and relevant magnetic field distribution. Most of these values were measured by optical emission spectroscopy or Langmuir probes [4] [34] [35]; the positive ion temperature and the negative ion emission rate from the Cs covered PG surface were theoretically estimated based on the Frank-Condon energy of hydrogen atoms $\left(E_{F C}=2.24 \mathrm{eV}\right)$ [38] and atomic hydrogen flux to the PG respectively [21]. The initial temperature of the surface produced NI is an educated guess value based on the energy of the hydrogen atoms colliding the plasma grid wall. It was found that production of NI at the plasma grid surface from the conversion of impinging atoms is dominant over conversion of positive ions [21] [39].

The initial bulk plasma covers the first $\sim 10 \mathrm{~mm}$ of the simulation domain and it is composed of $\mathrm{H}^{+}, \mathrm{H}_{2}{ }^{+}, \mathrm{H}_{3}{ }^{+}, \mathrm{Cs}^{+}, \mathrm{H}^{-}$and electrons. The particles are injected with a Maxwellian energy distribution using the typical temperatures listed in Table 1. The plasma neutrality in this region is maintained by re-injection of the same kind of particle if one leaves the simulation domain (extracted or destroyed by collision with walls). The particles are re-injected randomly in the region where initial plasma was assumed $(0<x<10 \mathrm{~mm})$.

Table 1. Main input plasma parameters for ONIX simulations based on the experimental data from BATMAN testbed.

\begin{tabular}{|l|c|}
\hline \multicolumn{2}{|c|}{ Densities of plasma species } \\
\hline $\mathrm{n}_{\mathrm{e}}$ & 1.8 or $1 \times 10^{17} \mathrm{~m}^{-3}$ \\
$\mathrm{n}_{\mathrm{H} \text {-(in bulk plasma) }}$ & 0.2 or $1 \times 10^{17} \mathrm{~m}^{-3}$ \\
$\mathrm{n}_{\mathrm{H}+}$ & $0.8 \times 10^{17} \mathrm{~m}^{-3}$ \\
$\mathrm{n}_{\mathrm{H} 2+}$ & $0.8 \times 10^{17} \mathrm{~m}^{-3}$ \\
$\mathrm{n}_{\mathrm{H} 3+}$ & $0.3 \times 10^{17} \mathrm{~m}^{-3}$ \\
$\mathrm{n}_{\mathrm{Cs}+}$ & $0.1 \times 10^{17} \mathrm{~m}^{-3}$ \\
$\mathrm{n}_{\mathrm{H}}$ & $1.0 \times 10^{19} \mathrm{~m}^{-3}$ \\
$\mathrm{n}_{\mathrm{H} 2}$ & $4.0 \times 10^{19} \mathrm{~m}^{-3}$ \\
\hline \multicolumn{2}{|c|}{ Temperatures of plasma species } \\
\hline $\mathrm{T}_{\mathrm{e}}$ & $2.0 \mathrm{eV}$ \\
$\mathrm{T}_{\mathrm{H}-\text { (volume) }}$ & $0.8 \mathrm{eV}$ \\
\hline
\end{tabular}




\begin{tabular}{|l|c|}
\hline $\mathrm{T}_{\mathrm{H}-\text { (surface) }}$ & $1.0 \mathrm{eV}$ \\
$\mathrm{T}_{\mathrm{H}+}$ & $0.8 \mathrm{eV}$ \\
$\mathrm{T}_{\mathrm{H} 2+}$ & $0.1 \mathrm{eV}$ \\
$\mathrm{T}_{\mathrm{H} 3+}$ & $0.1 \mathrm{eV}$ \\
$\mathrm{T}_{\mathrm{Cs}+}$ & $0.8 \mathrm{eV}$ \\
$\mathrm{T}_{\mathrm{H}}$ & $0.8 \mathrm{eV}$ \\
$\mathrm{T}_{\mathrm{H} 2}$ & $0.1 \mathrm{eV}$ \\
\hline \multicolumn{1}{|c|}{ Extraction potential } & 10 or $5 \mathrm{kV}$ \\
\hline H' emission rate from & from 33 to \\
Cs covered PG surface & $60 \mathrm{~mA} / \mathrm{cm}^{2}$ \\
\hline
\end{tabular}

The negative ions are self-consistently produced in the volume via the electron dissociative attachment collision to hydrogen molecules in the upper vibrational levels $(v>4)$ using the average calculated molecular densities from [40]. The negative ion production at the caesium covered plasma grid surface is introduced in the model by a NI emission flux. The optimum rate for the lowest value of the surface work function $(\sim 1.6 \mathrm{eV}[41])$ and clean surface of the PG was calculated by the 1D PIC simulation model BACON $\left(\sim 60 \mathrm{~mA} / \mathrm{cm}^{2}\right)$ [21]. A parametric study of this flux was performed for this paper representing different states of the Cs source conditioning in order to determine its influence on the formation of the negative potential well and the ion-ion plasma.

A full 3D realistic magnetic field distribution previously calculated by an external code was exploited for ONIX simulations [8]. This magnetic field was obtained taking into account both the filter and the electron deflecting field with the arrangement of the permanent magnets as installed in the BATMAN testbed. The aperture considered by ONIX is one of the central part of the LAG grid. The vertical and axial component of the magnetic field is inhomogeneous in range of one aperture [8] due to the deflecting field distribution one proving the necessity of using a $3 \mathrm{D}$ model for a realistic simulation of the addressed problem.

In order to speed up the calculation, the code is parallelized via the Message Passing Interface using full spatial domain and particle decomposition techniques [42]. The steady state condition with aforesaid parameters is typically reached after $\sim 2$ weeks of run using 20 CPUs. When more CPUs are involved in the simulation the total real computation time is not significantly improved. A possible explanation is that communication between processors limits drastically the parallel efficiency when high amount of CPUs is used or not optimal particle load balance that is a subject for future code optimization.

In order to be closer to the experimental conditions and perform more realistic simulations $\mathrm{Cs}^{+}$ions were introduced in the ONIX code. Due to their higher mass, the Cs ions stay much longer close to the PG surface and could reduce the depth of the virtual cathode. The $\mathrm{Cs}^{+}$density distribution along the simulation domain is shown in Fig. 3. The Cs ions as positively charged species are organized in the meniscus structure close to the plasma grid with a maximum in the plasma generation volume. The quasi steady state condition for $\mathrm{Cs}^{+}$is reached much later than for hydrogen ions, therefore density peaks fluctuations are still visible at the border on the initial plasma volume. A detailed analysis of the influence of $\mathrm{Cs}^{+}$ions on the surface produced NI transport will be given in the results section. 


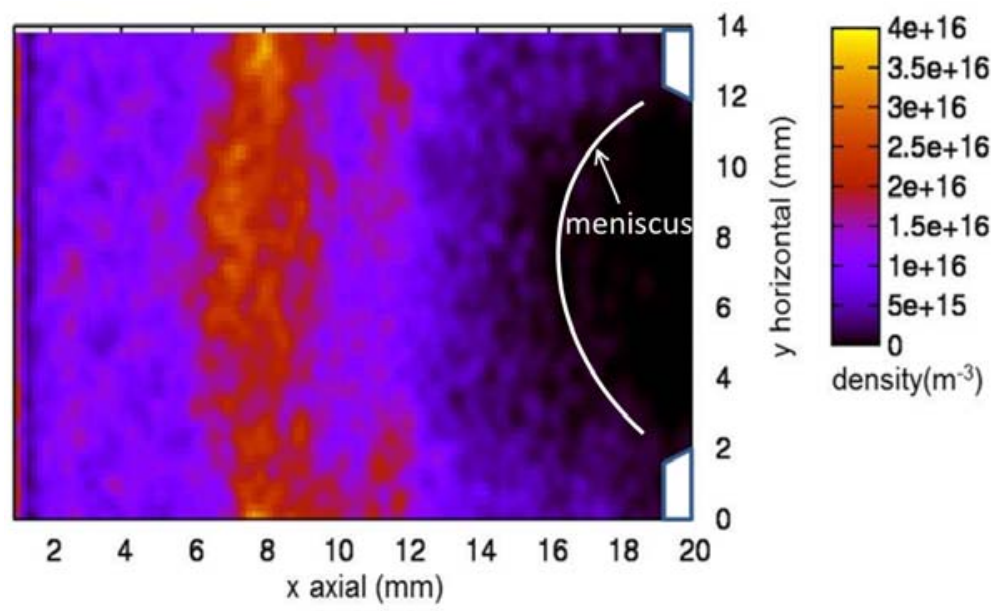

Fig. $3 \mathrm{Cs}^{+}$distribution in the simulation domain from the calculation using plasma parameters from Table 1 . The initial $\mathrm{Cs}^{+}$density is $10^{16} \mathrm{~m}^{-3}$.

Additionally introduced to the model was the bias potential applied to the plasma grid in order to decrease co-extracted electron current. The potential difference between the plasma and the grid could accelerate also NI towards the bulk plasma region contributing in the ion-ion plasma formation, which was impossible to obtain in self-consistent manner using previous code version [8]. In the experiments the bias potential is applied to the plasma grid with respect to the bias plate, which is connected to the source body. In the simulation model only a potential difference between the plasma potential and the PG is introduced. The potential of the plasma is at $0 \mathrm{~V}$, therefore, the plasma grid potential is set to values between $\mathrm{V}_{\text {bias }}=-10: 0 \mathrm{~V}$ in order to have in the code potential differences similar to the experiment.

Zoomed potential distributions along the simulation domain including different values for these plasma grid bias: $0 \mathrm{~V},-5 \mathrm{~V}$ and $-10 \mathrm{~V}$ are shown in Fig. 3. It can be seen that the bias potential applied to the plasma grid does not change the potential distribution in the bulk plasma region $(0 \leq x \leq 12 \mathrm{~mm})$. However, the bias potential slightly reduces the depth of the virtual cathode from $\sim 8 \mathrm{~V}$ at $0 \mathrm{~V}$ bias to $\sim 6 \mathrm{~V}$ at $-10 \mathrm{~V}$ bias. The influence of the bias potential on the NI transport from the plasma grid surface and the ion-ion plasma formation is discussed in the next section.

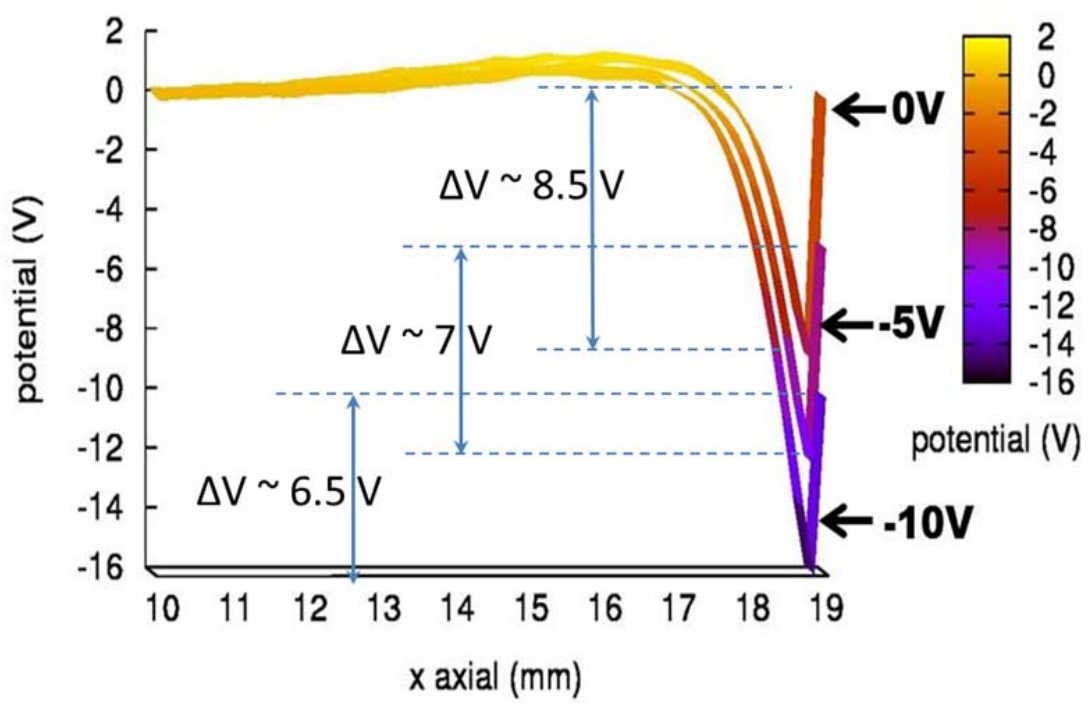

Fig. 4 Axial potential distribution in $x-z$ plane $(y=0.5 \mathrm{~mm})$ from the plasma side $(x=10 \mathrm{~mm})$ to the plasma grid $(x=19.1$ $\mathrm{mm}$ ) for three bias values of the plasma grid: (i) $0 \mathrm{~V}$, (ii) $-5 \mathrm{~V}$, and (iii) $-10 \mathrm{~V}$. All simulations were performed keeping constant the NI emission rate at $60 \mathrm{~mA} / \mathrm{cm}^{2}$ taking from [21]. 


\section{Results and Discussions}

\section{Meniscus formation}

The high positive extraction potential not only attracts negative hydrogen ions and electrons but also repels the positively charged particles back into the expansion chamber. The simulated particles are organized there in a semi-spherical structure called meniscus (Fig. 5). The meniscus can be also defined as the boundary between the plasma and the extracted beam, where the system potential is $\sim 0 \mathrm{~V}$. Using the new version of ONIX and as input the plasma and source parameters from BATMAN allows us to calculate more realistic meniscus distribution. The meniscus position and the deepness of its curvature (with respect to the PG plane at $x=19.1 \mathrm{~mm}$ ) plays an important role for the NI beam formation and the beam optics as it defines the velocity starting angle of the extracted particles. Moreover, the extraction of NI, independently of their production path (surface or volume), is governed by the meniscus shape, which in turn depends also of the local density of charged species.

In order to compare the meniscus shape for poor and optimum perveance condition (defined above) two simulations with extraction potential of 5 and $10 \mathrm{kV}$ were performed. Fig. 5 shows the $\mathrm{H}^{+}$density distribution for the calculations using $10 \mathrm{kV}$ (a) and $5 \mathrm{kV}$ (b). For both calculations the other plasma parameters were taken from Table 1. As it is expected, for the smaller extraction potential the meniscus moves towards the plasma grid plane due to the better plasma screening effect. In the case of $10 \mathrm{kV}$ extraction potential the deepest meniscus curvature point is located at a distance $\sim 1.6 \mathrm{~mm}$ from the beginning of the plasma grid plane (Fig. 5, a) and two onset points are placed at the distance $\sim 0.4 \mathrm{~mm}$ from the center of the PG $(x=21.1 \mathrm{~mm})$. When the extraction potential of $5 \mathrm{kV}$ is used the deepest meniscus curvature point is located at a distance $\sim 0.6 \mathrm{~mm}$ from the plasma grid plane with onset points at the distance $\sim 0.2 \mathrm{~mm}$ from the center of the PG (Fig. 6 , b). A similar behavior was detected in the simulations of the ITER-like CEA grid with aperture diameter of $14 \mathrm{~mm}$ [22] and in the simulations of the Japanese $\mathrm{H}^{-}$source used at JAEA [31]. However, the absolute value of the $\mathrm{H}^{+}$density in the vicinity to the meniscus is different in all these simulations. The higher density of the positive ions close to the plasma grid surface compensates better the negative space charge of the surface produced NI reducing thus the depth of the virtual cathode.

In spite of the small extraction potential $(5 \mathrm{kV})$ representing the optimum perveance the meniscus position is still penetrating into the diffusion chamber out of the plane of the plasma grid. In the simulations such meniscus shape with small positive ion density at the location of the meniscus onset points and without the plasma inside the plasma grid aperture $(19 \leq x \leq 21 \mathrm{~mm}$; $4 \leq y \leq 10 \mathrm{~mm}$; Fig. 5) provokes direct negative ions extraction from the surface produced NI.
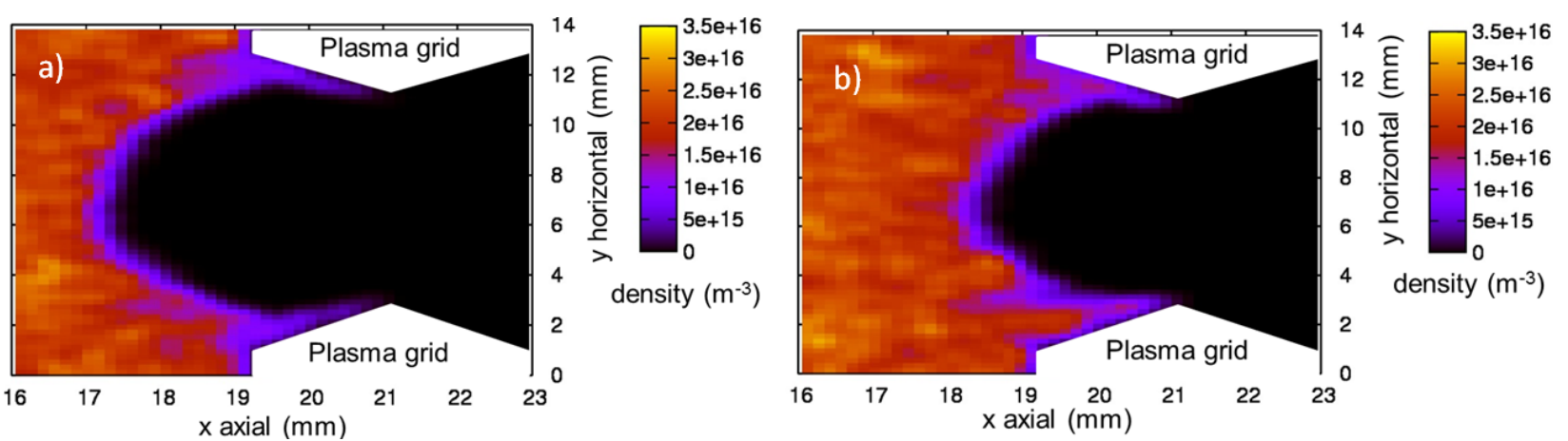

Fig. 5. $\mathrm{H}^{+}$density distribution close to the PG aperture in horizontal mid $x-y$ plane in the simulation of BATMAN LAG plasma grid aperture for the extraction potential $10 \mathrm{kV}$ (a) and $5 \mathrm{kV}(\mathrm{b})$ with the initial plasma parameters from the

Table 1: NI emission rate from PG surface $\sim 60 \mathrm{~mA} / \mathrm{cm}^{2} ; \mathrm{n}_{\mathrm{H}^{-}}=\mathrm{n}_{\mathrm{e}}=10^{17} \mathrm{~m}^{-3}$ in the bulk plasma region. 


\section{Evolution of the extracted NI and co-extracted electron current in different Cs conditioning states}

One of the features of the ONIX code is its ability to predict in addition to the total extracted NI and co-extracted electron currents, the origin of the extracted particles distinguishing between the NI production channels. Therefore, the simulations results could be benchmarked with experimental data in terms of the extracted current giving the relevance of each production channels. Fig. 6 shows the time evolution of the extracted NI (left side of the figure) and the electron current densities (right side of the figure) using as input parameter well Cs conditioning status explained above. This condition corresponds to the total NI density in the bulk plasma region $\mathrm{n}_{\mathrm{H}^{-}}=10^{17} \mathrm{~m}^{-3}$ where $20 \%$ is volume produced NI and $80 \%$ surface produced NI [25]. The NI emission rate from the PG surface is $\sim 60 \mathrm{~mA} / \mathrm{cm}^{2}$, corresponding to the maximum possible NI production rate from a clean Cs covered plasma grid surface [21]. The extraction potential $10 \mathrm{kV}$ is used in this simulation in order to find the maximum extractable NI current. The results using $5 \mathrm{kV}$ extraction potential corresponding to the optimal perveance will be presented below.

All currents are evaluated at the exit plane of the simulation domain at the distance $3.5 \mathrm{~mm}$ from the plasma grid back plane (Fig. 2). Fig. 6-left shows the extracted NI current densities from the different channels of the NI production. The extracted NI current density from the surface produced NI, which after their creation do not penetrate in the bulk plasma region but move directly towards the extraction grid through the meniscus onsets or without interaction with any of the plasma particles (direct extraction) due to the presence of the extraction potential and absence of the plasma in this region, is $\sim 23 \mathrm{~mA} / \mathrm{cm}^{2}$. The extracted NI current density from the negative ions initially located in the bulk plasma region is $\sim 7 \mathrm{~mA} / \mathrm{cm}^{2}$ and extracted NI current from the surface production channel, which move first towards the plasma bulk and afterwards extracted is $\sim 0.5 \mathrm{~mA} / \mathrm{cm}^{2}$.

The total extracted NI current density for such conditions is $\sim 30 \mathrm{~mA} / \mathrm{cm}^{2}$ (Fig. 6- right), being in very good agreement with experimental measurements performed at the BATMAN testbed for well Cs conditioning regime and similar plasma and source parameters [4] [35]. The coextracted electron current density is $\mathrm{j}_{\mathrm{e}} \sim 27 \pm 7 \mathrm{~mA} / \mathrm{cm}^{2}$ in steady state condition ( $>1.2 \mu$ s Fig. 6 -right), thus $\mathrm{j}_{\mathrm{e}} \approx \mathrm{j}_{\mathrm{H}}$, being also in good agreement with the experiment.
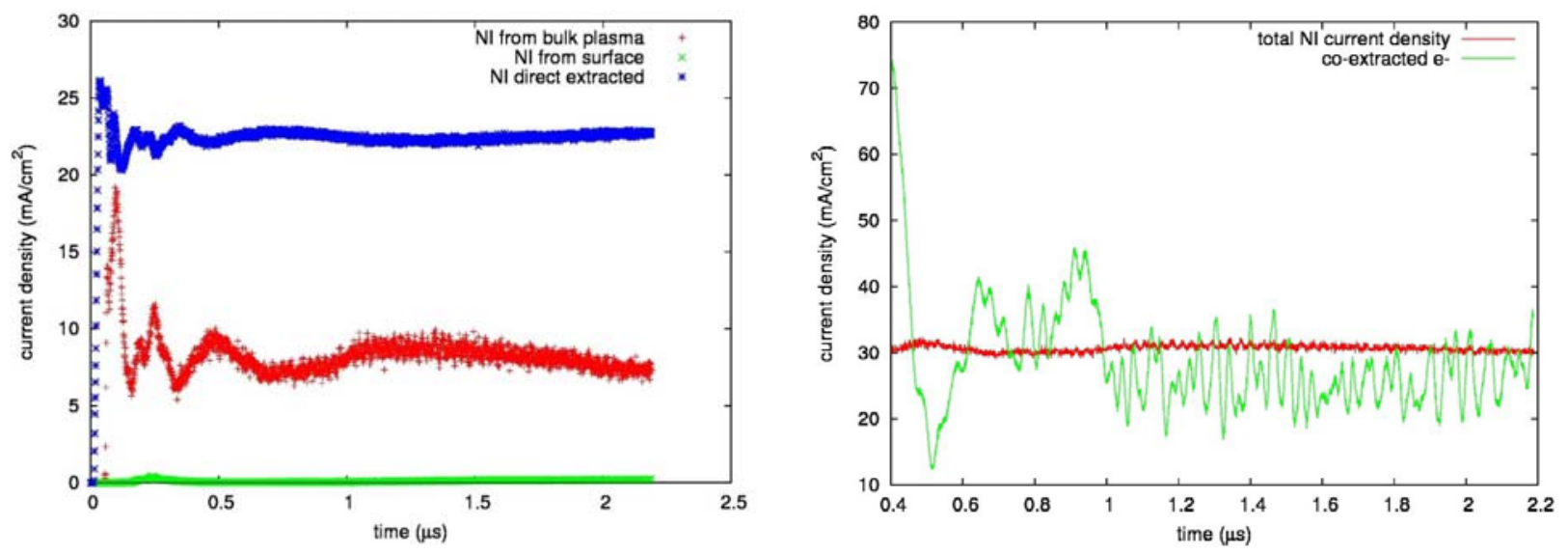

Fig. 6 Time evolution of the extracted NI current density from different production channels. The extracted potential of $10 \mathrm{kV}$ and $60 \mathrm{~mA} / \mathrm{cm}^{2} \mathrm{NI}$ emission rate were used in the simulation with the plasma parameters from the Table 1 and equal density of $\mathrm{H}^{-}$and electron in the bulk plasma region $\mathrm{n}_{\mathrm{H}^{-}}=\mathrm{n}_{\mathrm{e}}=10^{17} \mathrm{~m}^{-3}$.

In the simulation presented in Fig. 6 the maximum $\mathrm{H}^{-}$emission rate $\left(60 \mathrm{~mA} / \mathrm{cm}^{2}\right.$ [21]) was used representing the best theoretically possible Cs conditioning of the source. Decreasing this NI emission rate from the optimal value allows the simulation of different stages of the Cs conditioning 
process. Thus, a simulation with an emission rate of $40 \mathrm{~mA} / \mathrm{cm}^{2}$ was performed using the same plasma and source parameters as for the run presented in Fig. 6. The total extracted NI current density decreases reaching only $\sim 26 \mathrm{~mA} / \mathrm{cm}^{2}$, mostly due to the reduction of the direct NI extraction from the surface produced negative ions. The co-extracted electron current density increases slightly compared to the previous simulation $\mathrm{j}_{\mathrm{e}} \sim 29 \pm 7 \mathrm{~mA} / \mathrm{cm}^{2}$, reversing the $\mathrm{j}_{\mathrm{e}} / \mathrm{j}_{\mathrm{H}-}$ that becomes larger than 1. These results are again in good agreement with measurements performed at the BATMAN testbed [4] [35] assuming middle level of Cs conditioning.

In order to analyze the system performance for the good perveance condition two more simulations were performed with the same plasma parameters and NI emission rates $(60$ and 40 $\mathrm{mA} / \mathrm{cm}^{2}$ ) but with the extraction potential equals to $5 \mathrm{kV}$. For $60 \mathrm{~mA} / \mathrm{cm}^{2}$, in spite of using the optimum value for the $\mathrm{H}^{-}$emission rate, the extracted $\mathrm{NI}$ and co-extracted electron current densities both decrease down to $\mathrm{j}_{\mathrm{H}-}=\mathrm{j}_{\mathrm{e}}=\sim 20 \mathrm{~mA} / \mathrm{cm}^{2}$. Reducing the emission rate of $40 \mathrm{~mA} / \mathrm{cm}^{2}$ and keeping $5 \mathrm{kV}$ of extraction potential, the NI current density decreases even more reaching only $15 \mathrm{~mA} / \mathrm{cm}^{2}$, where $\sim 60 \%$ is due to the direct NI extraction and $\sim 40 \%$ to the NI present in the bulk plasma region. The co-extracted electron current stays at the same level $\sim 20 \mathrm{~mA} / \mathrm{cm}^{2}$. Such results are also in agreement with experimental measurements [4] in terms of total extracted currents.

From the simulations presented above one can derive that in present state of ONIX it shows that the direct NI extraction from the surface produced NI is dominant in all cases. In the optimum perveance condition $\left(5 \mathrm{kV}\right.$ extraction potential and $40 \mathrm{~mA} / \mathrm{cm}^{2} \mathrm{NI}$ emission rate) the results are closer to an equilibrated contribution between the direct NI extraction and the extraction from the bulk plasma volume. However, the NI emission rate being present in the experiment and the temperature of the NI produced at the plasma grid surface are still unknown. Most of the models use the temperature of $1 \mathrm{eV}$ taking into account the NI surface production process [17] and average energy of the incident hydrogen atoms and positive ions.

\section{Formation of the ion-ion plasma in the bulk plasma region}

The formation of an ion-ion plasma in the region of the extended boundary layer significantly impacts the meniscus structure, which in turn defines the shape of the negative ion beamlet. The previous version of the ONIX code [8] could not self-consistently reproduce the ion-ion plasma in the bulk region, which was observed in BATMAN [25]. In the following sections are analyzed and discussed the influence of the different source parameters (newly introduced in the code are the bias potential and the $\mathrm{Cs}^{+}$density; the NI emission rate and the initial temperature of the surface produced negative ions) on the deepness of the virtual cathode in front of the plasma grid that directly relies on the density of NI in the bulk plasma region.

In the simulations presented in the previous section the amount of NI in the bulk plasma was initially set to the density measured at BATMAN testbed for a good status of the Cs conditioning $\left(\mathrm{n}_{\mathrm{H}-}=10^{17} \mathrm{~m}^{-3}\right)$. It was measured that the volume density of NI increases by a factor of 5 from $\sim 2 * 10^{16} \mathrm{~m}^{-3}$ during the operation without $\mathrm{Cs}$ to $6-10 * 10^{16} \mathrm{~m}^{-3}$ after Cs vapor is injected in the source [25]. The NI density distribution in the bulk plasma region from the surface produced NI calculated by ONIX using the plasma parameters from Table 1 with $10 \mathrm{kV}$ extraction potential and $60 \mathrm{~mA} / \mathrm{cm}^{2}$ $\mathrm{NI}$ emission rate is shown in Fig. 7. The bias potential applied to the plasma grid and $\mathrm{Cs}^{+}$ions were not included in this calculation in order to show afterwards their influence on formation of the ionion plasma.

One can see that the NI density maximum hardly reaches $0.3 * 10^{14} \mathrm{~m}^{-3}-$ a result that is very low compared to measured values. Such disagreement is mostly due to high negative potential well in front of the plasma grid $(\sim 8 \mathrm{~V})$, which reduces the transport of the surface produced NI into the bulk plasma region. Only the negative ions with high kinetic energy could overcome this barrier making their initial temperature one of most critical parameters. As will be presented in the next sections, reduction of the depth of the negative potential well results in an increased NI density in the bulk plasma region and thus a better agreement of the ONIX simulation results with the experiment. 


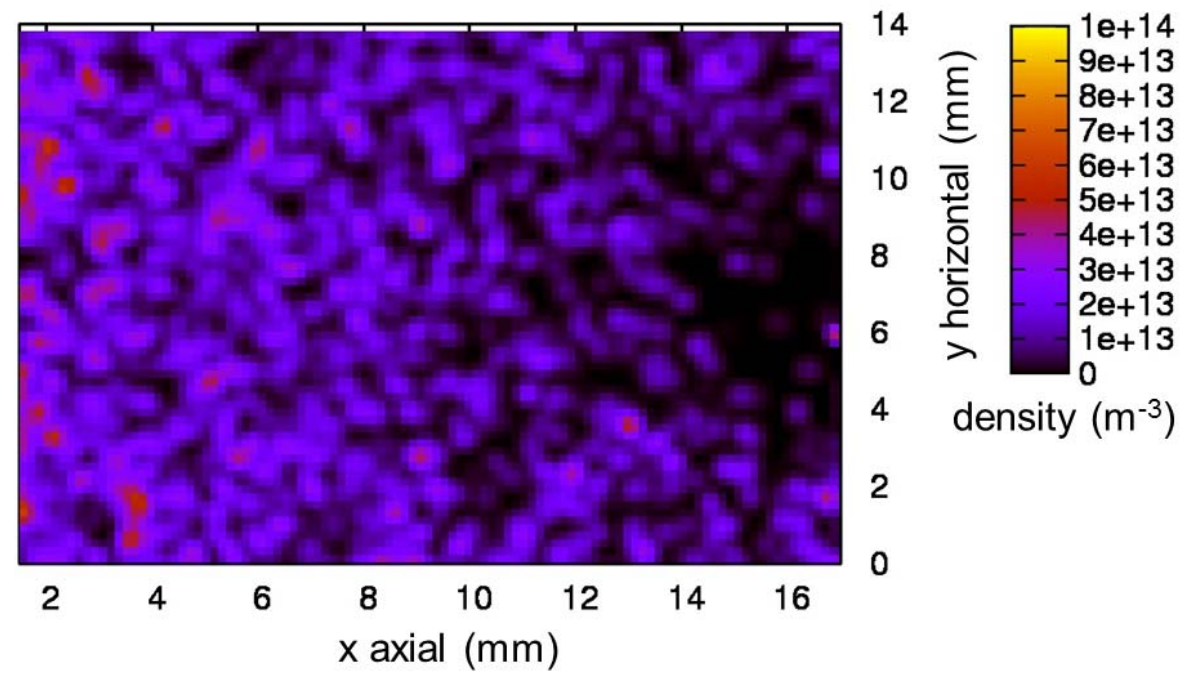

Fig. 7. Surface produced $\mathrm{H}^{-}$density distribution in horizontal mid $x-y$ plane along the bulk plasma region $(0 \leq x \leq 14 \mathrm{~mm}$, the PG front wall is placed at $x=19.1 \mathrm{~mm})$. NI emission rate $60 \mathrm{~mA} / \mathrm{cm}^{2}$; plasma parameters from Table 1 , extraction potential $10 \mathrm{kV}$, initial temperature of the surface produced $\mathrm{NI} 1 \mathrm{eV}$, plasma density $10^{17} \mathrm{~m}^{-3}$, without bias potential and $\mathrm{Cs}^{+}$ions.

\section{Influence of the bias potential on the depth of the virtual cathode}

As it was mentioned in section II, the recent version of ONIX was upgraded with a routine considering the bias potential applied to the plasma grid in order to study its influence on decreasing the depth of the virtual cathode and on the formation of an ion-ion plasma.

In the negative hydrogen ion sources under investigation [4] [6] the plasma grid is slightly positively biased $\left(\mathrm{V}_{\text {bias }}=0-20 \mathrm{~V}\right)$ in order to increase the flux of electrons towards the PG surface by reducing the repelling field of the sheath. A direct consequence is a reduction of the co-extracted electron current. The difference between the plasma potential and bias potential is in most of the cases about few volts $\left(\mathrm{V}_{\text {plasma }}-\mathrm{V}_{\text {bias }} \approx 1-5 \mathrm{~V}\right)[43]$.

The plasma potential in the ONIX simulations is $\sim 0 \mathrm{~V}$, thus all previous calculations with grounded $P G$ were represented experimental condition with $\mathrm{V}_{\text {plasma }}-\mathrm{V}_{\text {bias }} \approx 0 \mathrm{~V}$. Appling the slightly negative potential at PG surface (bias potential) will represent the potential difference of few volts making the simulation more similar to the experiments. Such bias potential could change the structure of the virtual cathode accelerating the surface produced NI towards the expansion chamber that will be investigated in this section. A parametric study of the bias potential was performed by means of the ONIX code using three bias potential values $\left(\mathrm{V}_{\text {bias }}=0 \mathrm{~V},-5 \mathrm{~V},-10 \mathrm{~V}\right)$ making the potential difference of $\mathrm{V}_{\text {plasma }}-\mathrm{V}_{\text {bias }}=0 \mathrm{~V}, 5 \mathrm{~V}, 10 \mathrm{~V}$ respectively (Fig. 4). It was found that the deepness of the virtual cathode only slightly decreases when a negative potential is applied at PG surface. In case without bias the potential well is $\sim-8 \mathrm{~V}\left(\Delta V_{\text {virtual cathode }}=8 \mathrm{~V}\right)$; at the bias potential $-5 \mathrm{~V}$ the potential well is $\sim-12 \mathrm{~V}\left(\Delta V_{\text {virtual cathode }}=7 \mathrm{~V}\right)$ and at the bias potential $-10 \mathrm{~V}$ the potential well is $\sim-16.5 \mathrm{~V}\left(\Delta V_{\text {virtual cathode }}=6.5 \mathrm{~V}\right)$. In order to test the extreme case when the bias potential is higher than plasma one the simulation with $\mathrm{V}_{\text {bias }}=10 \mathrm{~V}$ (i.e. $\mathrm{V}_{\text {plasma }}-\mathrm{V}_{\text {bias }}=-10 \mathrm{~V}$ ) was also performed. This positive bias potential increases the depth of the potential well almost on the same value with $\Delta V_{\text {virtual cathode }} \approx 17 \mathrm{~V}$.

Due to the small reduction of the virtual cathode depth (from $\sim 8 \mathrm{~V}$ to $\sim 6.5 \mathrm{~V}$ ) when the $-10 \mathrm{~V}$ bias potential is used the negative ion density in the bulk plasma region only slightly increases from $0.4^{*} 10^{14} \mathrm{~m}^{-3}$ in the case without bias to $0.7 * 10^{14} \mathrm{~m}^{-3}$ when the $-10 \mathrm{~V}$ bias is applied. However, the absolute value of the simulated NI density is still much different than the one measured in the experiment $\left(6-10^{*} 10^{16} \mathrm{~m}^{-3}\right)$. 
The negative potential well in front of the plasma grid can be reduced by the presence of the positive ions in this region due to the space charge compensation. The positive caesium ions due to their heavy mass stay much longer than positive hydrogen ions in vicinity to the plasma grid reducing even more efficient the depth of the virtual cathode. In order to test this idea and making the code more similar to the experimental condition the $\mathrm{Cs}^{+}$ions, which were included up to now only in 1D PIC code [21], were also introduced in the ONIX simulation.

The $\mathrm{Cs}^{+}$density in the BATMAN testbed (up to $1-5^{*} 10^{16} \mathrm{~m}^{-3}$ ) was determined by measuring the neutral Cs density [4] [34] [35] and then estimating the ionization degree of Cs ( $>90 \%$ [34]). As typical value a $\mathrm{Cs}^{+}$density of $10^{16} \mathrm{~m}^{-3}$ was used for the ONIX calculations described in the following. Initially, caesium ions are distributed inside the expansion chamber from the left border of the simulation domain till the plasma grid plane $(x=19.1 \mathrm{~mm})$. During the transitory simulation time (when the system is far from the steady state condition) an important amount of $\mathrm{Cs}^{+}$stays close to the grid surface reducing the negative sheath. However, with time the caesium ions are pushed back by the extraction potential towards the expansion chamber, where they - as the other positively charged species - are organized in the meniscus (Fig. 3). The transitory time in the calculation with $\mathrm{Cs}^{+}$ions is much longer than in previous simulations due to their heavy mass. In the quasi steady state regime only a small portion of the $\mathrm{Cs}^{+}$is still present close to the plasma grid decreasing the potential well only by $<10 \%$ for the source and the plasma parameters from Table 1 . A similar result was found earlier by a $1 \mathrm{D}$ code [21]. However, such small reduction of the virtual cathode depth causes a growing of the negative ion density in the bulk plasma region by $\sim 15 \%$. This was not the case in the 1D simulations mostly due to the different depth of the virtual cathode.

It is known that during long operation of the source caesiated walls can release Cs atoms that are immediately ionized in the plasma [44] due to their very low ionization threshold compared to hydrogen species. Hence, these $\mathrm{Cs}^{+}$ions can contribute more in space charge compensation reducing thus the negative potential well.

\section{Influence of the negative ion emission rate on the virtual cathode depth}

The production of negative ions from the conversion of hydrogen atoms or positive ions at the Cs covered plasma grid surface is introduced in the ONIX code and in other PIC models [21] [31] [40] as arbitrary uniformly distributed flux of NI with a given emission rate. However, the correct value of this flux is still unknown, because it depends not only on the impinging particle fluxes but also on the conversion yield for both processes (positive ion and neutrals conversions). As it was discussed above the maximum value of the NI emission rate $\left(60 \mathrm{~mA} / \mathrm{cm}^{2}\right)$ was theoretically estimated in [21] using the NI conversion yield for a perfectly clean surface with the lowest possible work function (1.4-1.7eV [41]). However, in the real experiments due to the presence of impurities and different stages of the Cs conditioning in the source the Cs work function is higher $(>2.2 \mathrm{eV}$ [45]), thus emission rate will be smaller. The goal of the following simulation is to determine the system behavior using NI fluxes that are much smaller than the maximum theoretically possible value.

Simulations with the NI emission rate twice smaller than optimum $\left(33 \mathrm{~mA} / \mathrm{cm}^{2}\right)$ were performed. The smaller emission rate has two effects on the NI extraction in the calculation using ONIX code. On the one hand it decreases the total extracted NI current density (due to the significant reduction of the amount of negative ion, which are directly extracted) but on the other hand it reduces also the deepness of the virtual cathode allowing more NI overcome it and penetrate in the bulk plasma increasing thus the NI density and the extracted current from this region. It was found that the depth of the virtual cathode decreases to $\sim 4 \mathrm{~V}$ for such emission rate increasing the negative ion density in the bulk region from the surface produced NI increases in $\sim 3$ times. However, the extracted NI current from this production channel is still low in comparison to the direct extraction and total extracted NI current density becomes lower than electrons $\left(\mathrm{j}_{\mathrm{H}}^{-}\right.$ $\approx 21 \mathrm{~mA} / \mathrm{cm} ; \mathrm{j}_{\mathrm{e}} \approx 28 \mathrm{~mA} / \mathrm{cm}$ ). 


\section{Influence of the plasma density on the depth of the virtual cathode}

It is generally known that the meniscus position depends (besides the extraction potential) on the plasma density close to the extraction region. More dense plasma more efficiently screens the external potential pushing the equipotential lines towards the extraction system. The meniscus follows in the same direction and is placed closer to the plasma grid. This basic meniscus behavior was also shown in ONIX simulation in the previous paper in order to validate the code [8]. Higher density of the positive ions at the meniscus boundary (onsets points) better compensate the negative space charge in front of the plasma grid reducing the depth of the virtual cathode. However, the efficiency of such decreasing is still unknown. Therefore, the parametric simulation of different plasma densities was performed.

Three plasma densities were used as input for the simulations: $\mathrm{n}_{\mathrm{e}}+\mathrm{n}_{\mathrm{H}-}=\sum_{i}^{3} H_{i}^{+}+\mathrm{Cs}^{+}=10^{17} \mathrm{~m}^{-3}$; $2 * 10^{17} \mathrm{~m}^{-3}$ and $4 * 10^{17} \mathrm{~m}^{-3}$. This increase of the plasma density corresponds to an increase of the RF power in the experiments. The equivalent depths of the potential well for these densities are $8 \mathrm{~V}, 7 \mathrm{~V}$ and $6 \mathrm{~V}$ respectively. The NI density in the bulk plasma region increases almost by a factor of 2 when a twice dense plasma is simulated. However, the extracted NI current density from this channel (NIs which are produced at PG surface first move towards the bulk plasma region and afterwards extracted) is still very low in comparison to the directly extracted or volume produced negative ions. This fact is correlated to a still low absolute value of the NI density in the bulk region from this production channel $\left(2 * 10^{14} \mathrm{~m}^{-3}\right)$.

\section{Influence of the initial energy of the surface produced NI on the ion-ion plasma formation}

The following are the plasma and source conditions most appropriate in order to achieve an ion-ion plasma in the ONIX simulations for BATMAN: $5 \mathrm{kV}$ extraction potential, $-10 \mathrm{~V}$ bias potential, $\mathrm{Cs}^{+}$ density $10^{16} \mathrm{~m}^{-3}$, NI emission rate $33 \mathrm{~mA} / \mathrm{cm}^{2}$ and bulk plasma density $2 * 10^{17} \mathrm{~m}^{-3}$. By using these parameters, the NI density in the bulk plasma region from the surface produced NI increases by about two orders of magnitude with respect to the simulation presented in Fig. 7 from $\sim 3^{*} 10^{13} \mathrm{~m}^{-3}$ to $\sim 5^{*} 10^{15} \mathrm{~m}^{-3}$. However, this result is still one order of magnitude below the experimental measured values $\left(5-10^{*} 10^{16} \mathrm{~m}^{-3}\right)$. Consequently, the extracted NI current from these ions is still relatively low $\left(<1 \mathrm{~mA} / \mathrm{cm}^{2}\right)$. Therefore, it was decided to also perform a parametric study of the initial energy of the surface produced NI. Releasing the NI with higher initial energy from the surface will allow them to cross the virtual cathode contributing efficiently to the bulk plasma.

Fig. 8 shows the negative ion density distribution in the bulk plasma region from the NI produced on the Cs covered plasma grid surface using the plasma and source parameters presented above but with an initial NI temperature of $5 \mathrm{eV}$. The total NI density in the plasma bulk reaches $\sim 5^{*} 10^{16} \mathrm{~m}^{-3}, 40 \%$ of which are produced in the volume and $60 \%$ at the PG surface. Such results are already in better agreement with experiments $\left(5-10 * 10^{16} \mathrm{~m}^{-3}\right)$. However, the precise initial surface produced NI temperature is not known, as it was discussed above, but $5 \mathrm{eV}$ surely is too high assuming that conversion of the hydrogen atoms with low impinging energy $(<1 \mathrm{eV})$ at Cs covered PG surface is dominant process for NI creation. Nevertheless, such simulation proves feasibility by means of the ONIX code to achieve the sufficiently high NI density in the bulk plasma region from the surface produced NI. This simulation result shows also an important increase of the extracted NI current from the ions produced at PG surface $\left(\sim 2 \mathrm{~mA} / \mathrm{cm}^{2}\right)$ and traversing the bulk plasma before being extracted. However, its contribution to the total extracted current density is still much lower compared to direct extraction. This issue is a subject for future studies. 


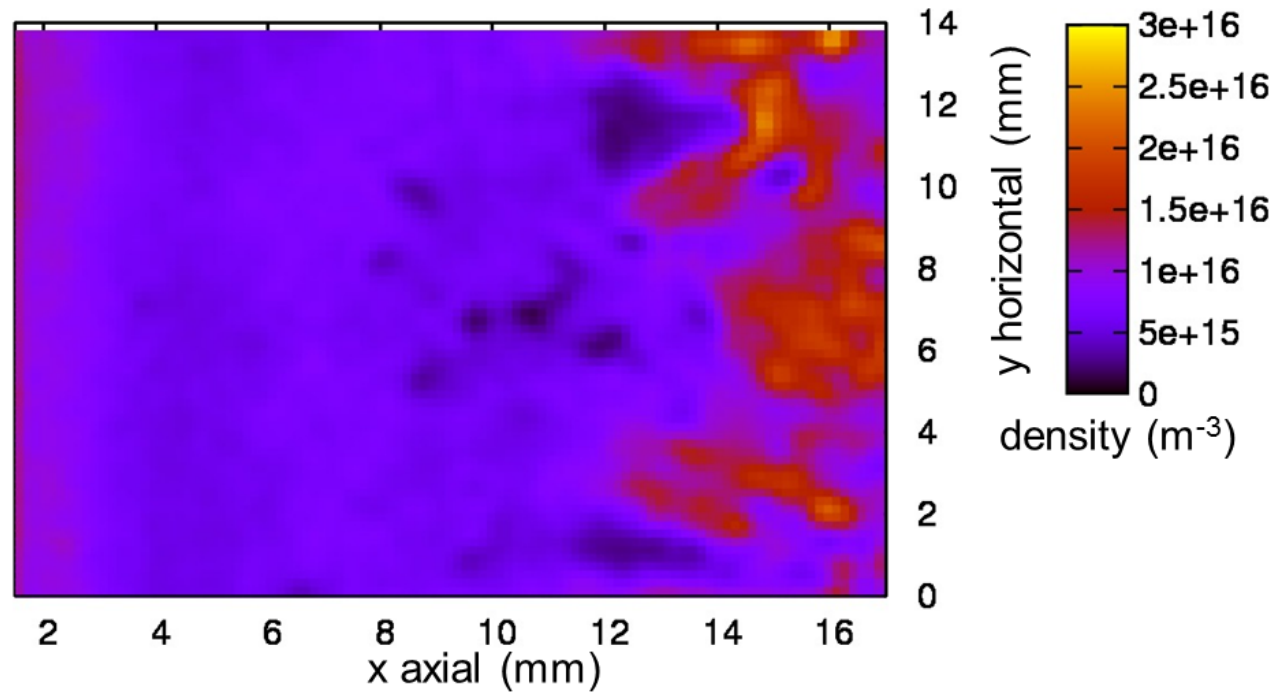

Fig. 8. Surface produced $\mathrm{H}^{-}$density distribution in horizontal mid $x-y$ plane along the bulk plasma region $(0 \leq x \leq 14 \mathrm{~mm}$, the PG front wall is placed at $x=19.1 \mathrm{~mm})$. NI emission rate $\sim 33 \mathrm{~mA} / \mathrm{cm}^{2}$; the extraction potential is $5 \mathrm{kV}$, surface produced NI initial temperature is $5 \mathrm{eV}$, plasma density $2 * 10^{17} \mathrm{~m}^{-3}$, bias potential is $-10 \mathrm{~V}$ and $\mathrm{n}_{\mathrm{Cs}+}=10^{16} \mathrm{~m}^{-3}$.

\section{Discussion towards the ion-ion plasma formation}

Implementing the plasma grid bias potential and $\mathrm{Cs}^{+}$ions in the ONIX simulations was fulfilled in order to perform more realistic simulation of the ITER prototype negative ion source extraction region. A parametric study of these parameters together with the NI emission rate, the plasma density and the value of the extraction potential resulted in NI density growth from the surface produced NI by more than two orders of magnitude in the bulk plasma region. Such achievement was obtained due to pushing the meniscus more closely to the plasma grid increasing thus the positive ion density in this region. This increased positive ion density in turn compensates more efficiently the negative space charge of the surface produced NI reducing the virtual cathode. In the best case the virtual cathode depth decrease from $\sim 8 \mathrm{~V}$ to the value of $3 \mathrm{~V}$. The meniscus in this case is located at the distance $\sim 0.5 \mathrm{~mm}$ from the front plane of the plasma grid improving the beam optics in comparison to the meniscus, which was calculated using previous version of the code [8] $(\sim 2 \mathrm{~mm}$ from PG). The importance of the direct NI extraction decreases simultaneously with reducing the NI emission rate, however its contribution to the total extracted NI current is still higher than extraction from the bulk plasma region.

The maximum obtained NI density is still lower than in the experiments. A possible explanation could be that NIs are produced not only at the aperture surface but also at the large flat surface of the bias plate beside the extraction aperture (or plasma grid) and their flux towards the center of the grid can be enough to reach sufficiently high density. Another reason can be absence in the ONIX simulation the electron losses due to their trapping along the magnetic field lines [31]. Reducing the negative space charge in the bulk plasma will push positive ions towards the plasma grid wall, where they more efficiently contribute to the reduction and even suppression of the virtual cathode.

\section{Conclusions and future plans}

Close collaboration with the experimentalists working at BATMAN testbed allow to improve the ONIX code and tested it afterwards. The physical aspects newly introduced to the code are the bias potential applied to the plasma grid and $\mathrm{Cs}^{+}$ions. With these novelties the simulations provide results which are in better agreement with experimental data.

The extracted NI and co-extracted electron currents from the ONIX simulations are in very good agreement with experimental measurements at BATMAN for both good $\left(\mathrm{V}_{\mathrm{ext}}=5 \mathrm{kV}\right)$ and poor $\left(\mathrm{V}_{\mathrm{ext}}=10 \mathrm{kV}\right)$ perveance conditions and a plasma density of $2 * 10^{17} \mathrm{~m}^{-3}$. In the simulation with good 
perveance the meniscus is located closer to the extraction system as it was expected from general physics. As a result, the depth of the virtual cathode in front of the plasma grid is decreased facilitating the surface produced NI to overcome it. However, the negative potential well is still deep enough $(\sim 7 \mathrm{~V})$ to limit the negative ion density in the bulk plasma region originating from the surface produced NI to $0.3 * 10^{14} \mathrm{~m}^{-3}$, which is about 3 orders of magnitude smaller than in the experimental measurements. It has been shown that the depth of the potential well can be decreased by introducing the plasma grid bias potential and the presence of $\mathrm{Cs}^{+}$. It was found that higher plasma density also reduces the virtual cathode effect due to a higher positive ion density in the vicinity of the plasma grid. Combining all these effects and assuming a lower NI emission rate than the one for minimum work function, i.e $33 \mathrm{~mA} / \mathrm{cm}^{2}$ instead of $60 \mathrm{~mA} / \mathrm{cm}^{2}$ the virtual cathode decreases to $\sim 3 \mathrm{~V}$ increasing thus the density of negative ion in bulk region in two orders of magnitude $\left(\sim 5^{*} 10^{15} \mathrm{~m}^{-3}\right)$, but it is still about 10 times smaller than in the experiments. Artificially increasing the starting temperature of NI to $5 \mathrm{eV}$ allows approaching the measured NI density $\left(\sim 6^{*} 10^{16} \mathrm{~m}^{-3}\right)$ in the bulk region with half of NI originating from the surface production channel. However, from the ONIX code the contribution of these ions to the total extracted NI current is still low in comparison to the directly extracted negative ions and it is thus still a subject for future study.

The electron confinement time and sources of the NI influxes from the source walls are candidates to be study next in order to reduce the discrepancy still present between the simulation and experimental results in terms of the NI density in the bulk plasma region. Furthermore the code will be adapted to simulate the ITER-like grid of the extraction system of the ELISE source.

\section{Acknowledgment}

Useful discussions with all members of ITED group at IPP are truly acknowledged. This work was done as a part of the Alexander von Humboldt fellowship.

\section{References}

[1] R. Hemsworth, A. Tanga and V. Antoni, Rev. Sci. Instrum., vol. 79, p. 02C109, 2008.

[2] R. Hemsworth, H. Decamps, J. Graceffa, B. Schunke, M. Tanaka, M. Dremel, A. Tanga, H.P.L. de Esch, F. Geli, J. Milnes, T. Inoue, D. Marcuzzi, P. Sonato, and P. Zaccaria, Nucl. Fusion, vol. 49, p. 045006, 2009.

[3] R. Nocentini et al., Fusion Engineering and Design, vol. 86, pp. 916-919, 2011.

[4] E. Speth, H. D. Falter, P. Franzen, U. Fantz, M. Bandyopadhyay, S. Christ, A. Encheva, M. Fröschle, D. Holtum, B. Heinemann, W. Kraus, A. Lorenz, Ch. Martens, P. McNeely, S. Obermayer, R. Riedl, R. Süss, R. Wilhelm A. Tanga, and D. Wünderlich, Nucl. Fusion, vol. 46, p. S220, 2006.

[5] U. Fantz, P. Franzen, W. Kraus, H. D. Falter, M. Berger, S. Christ-Koch, M. Fröschle, R. Gutser, B. Heinemann, C. Martens, P. McNeely, R. Riedl, E. Speth, and D. Wünderlich, Rev. Sci. Instrum, vol. 79, p. 02A511, 2008.

[6] B. Heinemann, U. Fantz, P. Franzen, M. Fröschle, M. Kircher, W. Kraus, C. Martens, R. Nocentini, R. Riedl, B. Ruf, L. Schiesko, C. Wimmer, D. Wünderlich, and the NNBI-Team., " Negative ion test facility ELISE - Status and first results.," 2013.

[7] P. Franzen et al., Nucl. Fusion, vol. 47, pp. 264-270, 2007.

[8] S. Mochalskyy, D. Wünderlich, B. Ruf, U. Fantz, P. Franzen and T. Minea, "On the meniscus formation and the negative hydrogen ion extraction from ITER NBI relevant ion source," Plasma Phys. Control. Fusion, vol. 56, p. 105001, 2014.

[9] A. Staebler et. al., "Development of a RF-driven ion source for the ITER NBI system," Fusion Engineering and Design, vol. 84, no. 2-6, p. 265-268, 2009.

[10] B. Heinemann, H. Falter, U. Fantz, P. Franzen, and M. Fröschle, Fusion Eng. Des., vol. 84, p. 915, 2009. 
[11] W. Kraus et al., AIP Conference Proceedings , vol. 1515, p. 129 , 2013.

[12] D. Wünderlich et al., Review of Scientific Instruments, vol. 84, p. 093102 , 2013.

[13] U. Fantz et al., Rev. Sci. Instrum., vol. 85, p. 02B305 , 2014.

[14] P. Franzen et al., Fusion and Energy Design, vol. in press, 2013.

[15] R. Janev, Elementary Processes in Hydrogen-Helium Plasma, 1987.

[16] M. Bacal, "Physics aspects of negative ion sources," Nucl. Fusion, vol. 46, pp. S250-S259, 2006.

[17] Y. Belchenko, Rev. Sci. Instrum., vol. 64, p. 1385 , 1993.

[18] Yu.I. Belchenko et al., Nucl. Fusion, vol. 14, p. 113, 1974.

[19] A. Hatayama, Rev. Sci. Instrum., vol. 79, p. 02B901, 2008.

[20] K. Miyamoto, S. Okuda, A. Hatayama, M. Hanada, A. Kojima, AIP conference proceedings, vol. 1515, pp. 22-30, 2013.

[21] D. Wünderlich, R. Gutser and U. Fantz, Plasma Sources Sci. Technol., vol. 18, p. 045031, 2009.

[22] S. Mochalskyy, A.F. Lifschitz and T. Minea, J. Appl. Phys., vol. 111, p. 113303, 2012.

[23] F. Taccogna, P. Minelli and S. Longo, Plasma Sources Sci. Technol., vol. 22, p. 045019, 2013.

[24] R. McAdams, A. J. T. Holmes, D. B. King and E. Surrey, Plasma Sources Sci. Technol. , vol. 20, p. 035023, 2011.

[25] M. Berger, U. Fantz, S. Christ-Koch and NNBI Team, Plasma Sources Sci. Technol., vol. 18, p. 025004, 2009.

[26] K. Tsumori et al., Rev. Sci. Instrum., vol. 83, p. 02B116, 2012.

[27] K.Ikeda, H.Nakano, K.Tsumori, M.Kisaki, K.Nagaoka, M.Osakabe, Y.Takaeiri and O.Kaneko, New Journal of Phys., vol. 15 , p. 103026, 2013.

[28] A. Hatayama, T. Shibata, S. Nishioka, M. Ohta, M. Yasumoto, K. Nishida, T.Yamamoto, K. Miyamoto, A. Fukano and T. Mizuno, Rev. Sci. Instrum. , vol. 85, p. 02A510, 2014.

[29] J. P. Boeuf, G. J. M. Hagelaar, P. Sarrailh, G. Fubiani and N. Kohen, Plasma Sources Sci. Technol., vol. 20, p. 015002, 2011.

[30] S. Mochalskyy, A. F. Lifschitz, and T. Minea,, Nucl. Fusion, vol. 50, p. 105011, 2010.

[31] K. Miyamoto, S. Okuda, S. Nishioka, and A. Hatayama, J. Appl. Phys., vol. 114, p. 103302 , 2013.

[32] S. Lishev et al., Conf. Proc. ICIS 2013, Chiba, Japan,, vol. in press, 2013.

[33] R Gutser et al., Plasma Phys. Control. Fusion, vol. 51, p. 045005, 2009.

[34] U. Fantz et al., "Diagnostics of the cesium amount in an RF negative ion source," Fusion Engineering and Design, vol. 74, p. 299-303, 2005.

[35] U. Fantz et al., "Spectroscopy—a powerful diagnostic tool," Nucl. Fusion, vol. 46, p. S297S306, 2006.

[36] R. Courant, K. Friedrichs, and H. Lewy, Math. Ann. , vol. 100, p. 3274, 1928.

[37] P. Franzen et al., Nucl. Fusion, vol. 51, p. 073035, 2011.

[38] U. Fantz and D. Wünderlich, "A novel diagnostic technique for $\mathrm{H}^{-}\left(\mathrm{D}^{-}\right)$densities in negative hydrogen ion sources," New Journal of Physics, vol. 8, p. 301, 2006.

[39] D. Wünderlich, L. Schiesko, P. McNeely, U. Fantz, P. Franzen, and the NNBI-Team, Plasma Phys. Control. Fusion, vol. 54, p. 125002, 2012.

[40] F. Taccogna et al., IEEE TRANSACTIONS ON PLASMA SCIENCE,, vol. 36, no. 4, 2008.

[41] W. G. Graham, in Proceedings of the Second International Symposium on the Production and Neutralization of Negative Hydrogen Ions and Beams, Brookhaven National Laboratory, Upton, New York, 1980. 
[42] M. Snir, MPI - The Complete Reference, Volume 1, The MPI Core (second edition), Cambridge: MA:MIT Press, 1998.

[43] L. Schiesko, private communication.

[44] R. Gutser, D. Wünderlich, U. Fantz and the N-NBI Team, "Dynamics of the transport of ionic and atomic cesium in radio frequency-driven ion sources for ITER neutral beam injection," Plasma Phys. Control. Fusion, vol. 53, p. 105014, 2011.

[45] R. Gutser, C. Wimmer, and U. Fantz, "Work function measurements during plasma exposition at conditions relevant in negative ion sources for the ITER neutral beam injection," Review of Scientific Instruments, vol. 82, p. 023506, 2011. 